\title{
Estimation of the strain field from full-field displacement noisy data
}

\section{Comparing Finite Elements Global Least Squares and po- lynomial Diffuse Approximation}

\author{
S. Avril ${ }^{*}-$ P. Feissel ${ }^{* *}-$ F. Pierron* - P. Villon** \\ * Laboratoire de Mécanique et Procédés de Fabrication \\ Ecole Nationale Supérieure d'Arts et Métiers \\ BP 508 rue Saint Dominique 51006 CHÂLONS EN CHAMPAGNE \\ ** Laboratoire Roberval de Mécanique, UTC, \\ BP 20529 rue Personne de Roberval 60205 COMPIEGNE
}

\begin{abstract}
RÉSUMÉ. Cette étude s'intéresse à la reconstruction de champs de déformation à partir de mesures de champs de déplacements bruités. Deux approches sont étudiées : l'une globale s'appuyant sur des Approximations Éléments Finis (AEF) et l'autre locale s'appuyant sur l'Approximation Diffuse $(A D)$. Ces deux approches sont comparées sur un cas test considéré comme diffcile (essai de traction sur éprouvette trouée). L'AD donne des résultats plus stables, mais coûte plus cher en temps de calcul. Finalement, un contrôle localisé de l'effet de filtrage des deux approaches est proposé en perspectives pour une amélioration prochaine de la reconstruction. ABSTRACT. In this study, the issue of deriving strain fields from corrupted full-field displacement data is addressed. Two approaches are proposed, a global one based on Finite Element Approximation (FEA) and a local one based on Diffuse Approximation (DA). Both approaches are compared on a case study which is supposed difficult (open-hole tensile test). DA provides more stable results, but is more computer expensive. Eventually, it is proposed to monitor locally the filtering effect of both approaches, the prospects being an impending improvement of the reconstruction for both approaches.

MOTS-CLÉS : mesures de champs, dérivation numérique, incertitude de mesure, identification. KEYWORDS: full-field measurements, numerical differentiation, measurement uncertainty, identification.
\end{abstract}




\section{Introduction}

The recent development of digitized full-field displacement measurements opens new ways of characterizing materials in solid mechanics (Kobayashi, 1993). However, for most of the users of these techniques the strain fields rather than the displacement fields provides a real insight in the physics of the material at different scales. Therefore, except for the techniques which provide directly the displacement derivatives, it is necessary to differentiate the data. When the gradients of the displacement fields are relatively low, for example when the materials still behave elastically, the small errors for measurement values may induce a large error to the computed derivative (Geers et al., 1996). So the key work is to develop a stable algorithm, in which it is possible to quantify explicitly the effects induced by noise differentiation.

A large number of algorithms can be found in the literature. A survey of these methods is briefly presented in (Wei et al., 2006). The most common approach is the finite difference method (Wei et al., 2006). Simple and effective with precise data, it is implemented in most of the softwares providing full-field displacement data. However, when the level of noise is significant, filtering is required.

One widely used way of doing this filtering consists in interpolating or approximating the data using smooth basis functions. The differentiation of the data is turned into the differentiation of the basis functions. For a given basis of functions, the regularization parameter is tied to the number of functions used in the basis. A good compromise between the faithfulness of the reconstruction (obtained with a large number of basis functions) and the efficiency of the low pass filtering (obtained with a small number of basis functions) has to be found.

However, a good choice of the basis functions is essential (Hickernell et al., 1999). The approximation can be defined either globally (Lira et al., 2004), or locally (Cleveland et al., 1995). Previous studies (Pierron et al., 2007) showed that a global polynomial basis leads to parasitic oscillations in the reconstruction when the degree of polynomials is too large. Indeed, because of the global aspect of this type of bases, local artifacts in the data affect the whole reconstruction. Accordingly, it seems that basis functions that have limited interactions between each other would suit better.

Two approaches fulfilling this requirement have been chosen and this paper is aimed at comparing them. The first approach is based on global least-squares minimization using Finite-Element shape functions as the basis functions (FEA) (Feng et al., 1991). The second approach is based on local weighted least-squares minimization using a polynomial diffuse approximation (DA) (Nayroles et al., 1991). The regularization/precision parameter is the mesh size for the first approach, the span of the weighting function in the second one.

In a first section, the framework and the principle of the proposed approaches are presented. Then, their efficiency is compared using modelled data. Eventually, the approaches are applied onto real data for solving a problem of damage detection. 


\section{Framework and presentation of the approaches}

\subsection{Framework and notations}

The proposed approaches are aimed at deriving strain fields from a set of full-field displacement measurements. These measurements are derived from the deformation of a pattern bonded onto the investigated solid, for instance using digital image correlation (Schreier et al., 2002) or fringe analysis (Surrel, 1994). Let us consider a given zone $\Omega$. The input data for deriving strain fields across $\Omega$ are therefore the displacements in both directions given at the nodes (called pixels) of a regular grid, whose coordinates are denoted $\underline{x}_{i}$. In the proposed examples, this grid is made of $N=168 \times 224$ pixels. These measurements are denoted as :

$$
\underline{\tilde{u}}\left(\underline{x}_{i}\right)=\underline{u}_{e x}\left(\underline{x}_{i}\right)+\delta \underline{u}\left(\underline{x}_{i}\right) \quad, \forall i \in[1, N]
$$

where $\delta \underline{u}$ represents the measurement error and $\underline{u}_{e x}$ is the exact mechanical field. The objective is to derive the gradients of $\underline{u}_{e x}$ for obtaining the exact strains.

In the following sections, the data are modelled or measured onto a plate tested in an open-hole tensile configuration, which is a difficult case with large strains concentrated near the hole (Balaco de Morais, 2000). The modelled data are obtained with a reference calculation (numerical example) in order to evaluate properly the reconstruction errors. The measured data are obtained on a real test carried out onto a glass/epoxy composite laminate. In the case of modelled data, $\underline{u}_{e x}$ and $\delta \underline{u}$ are known.

Since the reconstruction operator is linear (see 3.2 , the reconstructed field $\underline{u}_{a p}(\underline{x})$, at any $\underline{x}$, can be split up into three parts as follows :

$$
\underline{u}_{a p}(\underline{x})=\underline{u}_{e x}(\underline{x})+\delta \underline{u}_{k}(\underline{x})+\delta \underline{u}_{b}(\underline{x})
$$

where $\delta \underline{u}_{k}(\underline{x})$ corresponds to the error due to the approximation of the exact field and $\delta \underline{u}_{b}(\underline{x})$ is the error due to the noise contained in the measurements.

Due to the linearity of the differentiation operator, the reconstructed strains can be similarly split up in three terms.

\subsection{First approach : Global Least Squares / Finite Element approximation}

A first group of methods consists in using global least-squares minimization over the whole measurement zone $\Omega$. The choice of the basis functions on which the measurements will be projected will affect the regularity and the precision of the reconstruction. FEA (Feng et al., 1991), where the basis functions only have very low interactions between each other, is chosen in this study because it limits reconstruction oscillations as the precision increases, contrarily to polynomial basis functions (Pierron et al., 2007). The regularization parameter is hence the mesh size of the FEA.

The reconstructed displacement field is written like this :

$$
u_{a p}(\underline{x})=[\Phi(\underline{x})] U
$$


where $[\Phi(\underline{x})]$ is the matrix of the shape functions and $U$ is the vector of the nodal displacements. In our examples, the elements are triangles with linear shape functions.

$U$ is found as the solution of the following minimization :

$$
\min _{U} \sum_{i=1}^{N}\left(u_{a p}\left(\underline{x}_{i}\right)-\tilde{u}\left(\underline{x}_{i}\right)\right)^{2}
$$

The minimization problem [4] leads to a linear system to be solved, yielding $U$.

The strain field is directly derived from $u_{a p}(\underline{x})$, by differentiating the shape functions. In order to keep an approximated strain field described with the same shape functions, $\varepsilon_{a p}(\underline{x})=[\Phi(\underline{x})] E$, the strain field is projected onto the same basis of functions. Its nodal values are the solution of the following global least-squares problem :

$$
\min _{E} \int_{\Omega}\left(\varepsilon_{a p}(\underline{x})-[B(\underline{x})] U\right)^{2} d S
$$

where $[B(\underline{x})]$ is the symmetric gradient of $[\Phi(\underline{x})]$.

\subsection{Second approach : Diffuse Approximation / polynomial approximation}

A second group of methods is based on the use of local regression (Cleveland et al., 1995). Here, DA (Nayroles et al., 1991) is chosen with polynomial basis functions, various degrees being tested. As this approach is based on weighted least-squared, a key point is the span of the weighting function, denoted $R$. This parameter can be tuned to obtain the best regularization/precision compromise. As presented below, the (diffuse) derivatives are directly derived from the measurements with this approach.

DA enables to define a continuous field from a discrete number of data points. Given $p(\underline{x})$ a vector of basis functions, the following two variable function is introduced:

$$
v(\underline{x}, \underline{y})=p(\underline{y}-\underline{x})^{T}\{a(\underline{x})\}
$$

First $a(\underline{x})$ is sought as the solution of a local weighted least-square across the weighted neighborhood of $\underline{x}$, with respect to the $\underline{y}$ variable :

$$
\min _{a(\underline{x})} \frac{1}{2}(P\{a\}-\widetilde{U})^{T} W(P\{a\}-\widetilde{U})
$$

where $W$ is the diagonal matrix of the weighting function evaluated at each data point :

$$
W=\operatorname{diag}\left(\left[\begin{array}{c}
w\left(\underline{x}, \underline{x}_{1}\right) \\
\cdots \\
w\left(\underline{x}, \underline{x}_{N}\right)
\end{array}\right]_{i \in V(\underline{x})}\right)
$$


$w\left(\underline{x}, \underline{x}_{i}\right)$ can be any function defined over a bounded domain. The bounded domain requirement is aimed at keeping the local character of the reconstruction. Here, the weighting function is defined as :

$$
w\left(\underline{x}, \underline{x}_{i}\right)=w_{r e f}\left(\frac{x-x_{i}}{R_{x}}\right) w_{r e f}\left(\frac{y-y_{i}}{R_{y}}\right)
$$

where $w_{\text {ref }}$ is a dimensionless function whose derivative zeroes at 0 and 1 (this aspect ensures continuity up the the first derivative of the reconstructed fields); here $R_{x}$ and $R_{y}$ are chosen independant of $\underline{x}_{i}$ but may depend on $\underline{x}$.

$P$ and $\widetilde{U}$ in [7] concatenate respectively the basis functions and the data for all $\underline{y} \in V(\underline{x})$.

Then, the approximate field is defined as : $u_{a p}=v(\underline{x}, \underline{x})$, therefore, if the basis is composed of monomials up to at least degree 1 , one has :

$$
a_{2}(\underline{x})=\frac{\delta u}{\delta x}(\underline{x}) \quad \text { and } \quad a_{3}(\underline{x})=\frac{\delta u}{\delta y}(\underline{x})
$$

This reconstruction can be applied to each component of the displacement field $\underline{\tilde{u}}\left(\underline{x}_{i}\right)$. The strain fields can be deduced subsequently from the first order diffuse derivatives. Let us finally mention that this approach implies the resolution of problem [7] at each evaluation point. This means that one has to choose the evaluation points. Here, they are chosen as coincident with the pixels where data are provided. Accordingly, the reconstruction is evaluated through the discrete grid of the points $\underline{x}_{i}, i \in[1, N]$.

\section{Understanding and illustrating the methods with a numerical example}

\subsection{Numerical data and strain reconstruction}

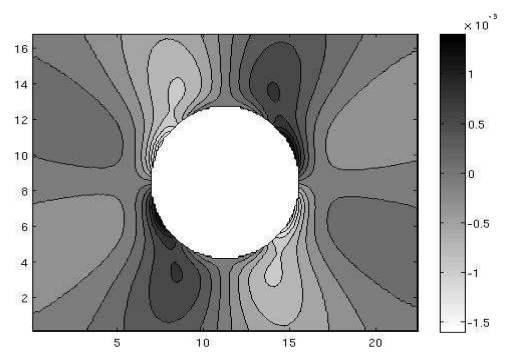

Figure 1. Exact shear strain fi eld to be reconstructed

In this section, the two methods presented in section 2 are applied to data modelled by a numerical simulation. A FE simulation of the tensile response of an elastic plate 
with a hole is thus performed. The obtained displacement field is then used to create a grid of data points and a white noise, with a realistic 5\% standard deviation, is added in order to represent the perturbation on the measurements. The strain is reconstructed from these measurements. At first, the strain reconstruction is illustrated on the shear strain field $\varepsilon_{X Y}$. In order to compare visually the quality of the reconstruction, the exact field is plotted in Figure 1] Figures 2 and 3 show the shear strain field reconstructed by both approaches for two different regularization parameters (mesh size or span of the weighting function) in each case. Let us remark that the larger the mesh size or span of the weighting function, the smoother the results. But the reconstructed field appears smoother with DA since the FEA first derivative is only $C^{0}$ at the edges of the elements, rending almost visible the mesh. In both cases, the approximation error

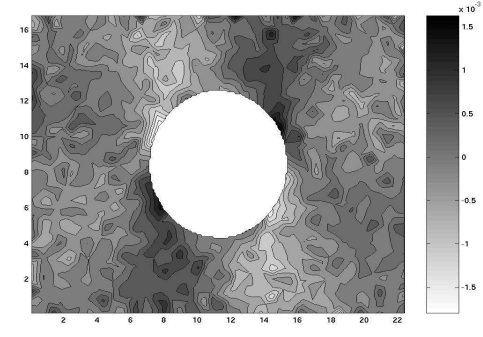

(a) Mesh size $: h=5$ pixels

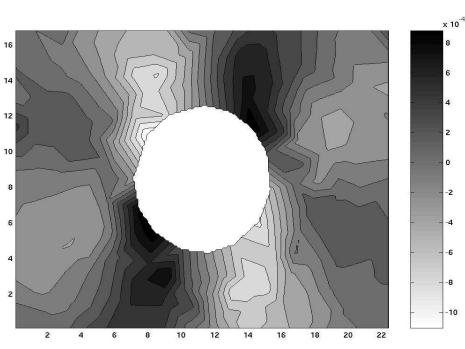

(b) Mesh size : $h=12$ pixels

Figure 2. Reconstructed shear strain fi eld $\mathbb{X}_{Y}$ with the FEA approach

$\delta \varepsilon_{k}$ remains quite small and is smaller in the DA approach. This will be confirmed in section 4.1

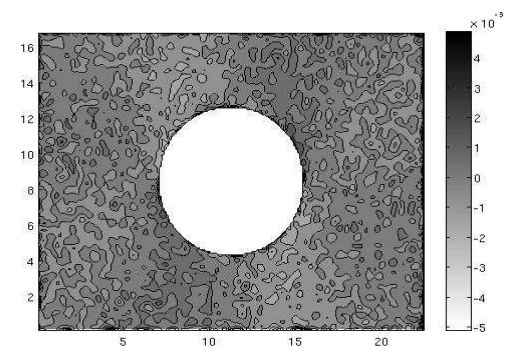

(a) Weight $\operatorname{span} R=7$ pixels

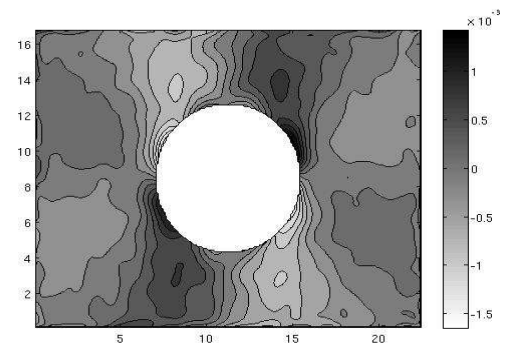

(b) Weight $\operatorname{span} R=18$ pixels

Figure 3. Reconstructed shear strain fi eld $母_{Y}$ with the DA method 


\subsection{Reconstructing operator}

As explained in section 2 the two proposed methods leads to the resolution of linear problems, which means that the reconstruction operators are linear. The first consequence is that, in the additive decomposition [2], the $\delta \underline{u}_{k}$ term corresponds to the reconstruction of the noise alone and the $\delta \underline{u}_{b}$ term is related to the reconstruction of the exact field. One can therefore study them separately.

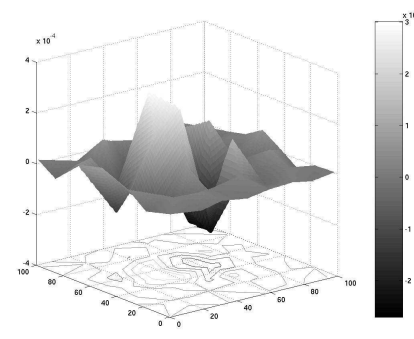

(a) Finite Elements method

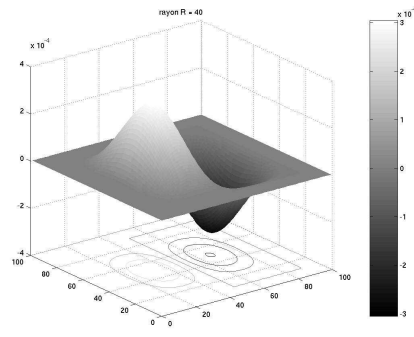

(b) Diffuse Approximation method

Figure 4. Green's function of the fi rst order derivative reconstruction operator

The second point is that one can define the Green's functions of the reconstructing operator. The Green's function at point $\underline{x}_{i}$ can be obtained as the reconstructed field with measurements such as : $\tilde{u}\left(\underline{x}_{j}\right)=\delta_{i j}$, with $\delta_{i j}$ the Kronecker function. As shown in Figure 4 the Green's functions confirm that both approaches only have a local domain of influence. In the FEA case, the effect of the mesh is visible.

\section{Numerical comparison of the methods}

\subsection{Global reconstruction error}

In this section, we propose to compare the two reconstruction approaches from a quantitative point of view. Consequently, one has to choose a criterium for evaluating the quality of the reconstruction. Since the reconstruction is applied to modelled data, this criterium is defined as a quadratic distance between the reconstructed strain field and the exact one :

$$
e_{\varepsilon}=\left\langle\sqrt{\left(\varepsilon_{X X}^{a p}-\varepsilon_{X X}^{e x}\right)^{2}+2\left(\varepsilon_{X Y}^{a p}-\varepsilon_{X Y}^{e x}\right)^{2}+\left(\varepsilon_{Y Y}^{a p}-\varepsilon_{Y Y}^{e x}\right)^{2}}\right\rangle_{\Omega_{m}}
$$

where $\langle\bullet\rangle_{\Omega_{m}}$ is the average of the data over region $\Omega_{m} . \Omega_{m}$ can be either the whole measurement zone $\Omega$ or a smaller zone around the hole where large gradients occur.

In Figure 5] error [11] is compared in various cases, for noisy and noiseless data, from strain fields reconstructed with the FEA approach and the DA approach. Within 


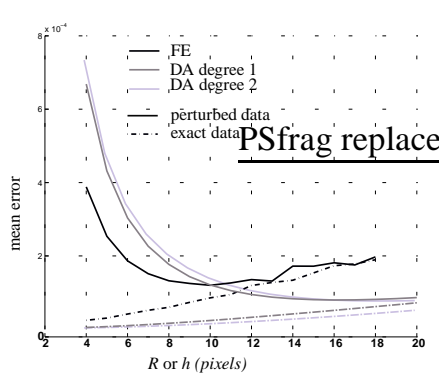

(a) Global error $e_{\varepsilon}$

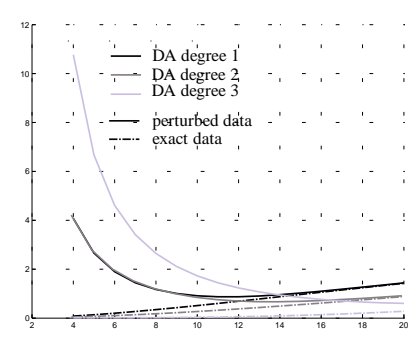

(b) Error $e_{\varepsilon}$ near the hole

Figure 5. Reconstruction error with respect to the characteristic size

the latter, degrees of the polynomial basis, ranging from 1 to 3 , are also compared. As expected, the perturbation error decreases as the mesh size or span of the weighting function increases, whereas the approximation error increases. One can notice that the FEA approach has a larger filtering effect than DA, but induces larger approximation errors, even when the basis functions of DA are of the same degree (degree 1). This can be explained by the larger size of the domain of influence of the FEA reconstruction operator.

In the considered example, the average of the approximation error obtained with DA remains small. The error at the vicinity of the hole for various degrees of the function basis used in DA is shown in Figure [5(b)] One observes that the approximation decreases as the degree increases, and the perturbation error increases. Nevertheless, degree 2 in our case is a good trade off between the filtering effect and the faithfulness of the reconstruction. From the curves plotted in Figure 5(b), it should also be emphasized that the approximation error depends on the zone. Therefore, it would be interesting to use mesh sizes or spans of the weighting function that vary spatially, depending on the zones. In order to do that, one has to define a criterium controlling the local mesh sizes or spans of the weighting function, for example a criterion based on the signal to noise ratio. Implementation of this feature is currently on-going.

\subsection{VFM-based stiffness identification using the reconstructed strains}

According to the basic equations of the Virtual Fields Method (VFM) (Grédiac et al., 2006), the Poisson's ratio of the material should verify the following equation :

$$
\mathrm{v}=-\frac{\int_{\Omega}\left(\varepsilon_{x x} \varepsilon_{x x}^{*}+\varepsilon_{y y} \varepsilon_{y y}^{*}+2 \varepsilon_{x y} \varepsilon_{x y}^{*}\right) d S}{\int_{\Omega}\left(\varepsilon_{x x} \varepsilon_{y y}^{*}+\varepsilon_{y y} \varepsilon_{x x}^{*}-2 \varepsilon_{x y} \varepsilon_{x y}^{*}\right) d S}
$$


where $\varepsilon^{*}$ is the strain field derived from a virtual displacement field $\underline{u}^{*}$ chosen across $\Omega$ in such a way that $u^{*}=0$ on $\partial \Omega$. The components $\varepsilon_{x x}, \varepsilon_{y y}$ and $\varepsilon_{x y}$ are the experimental strain fields reconstructed from the data. Because of data corruption, equation [12] is not satisfied in practice. Errors exist, but they can be minimized by choosing an optimal virtual field, denoted $\underline{u}^{* *}$, according to the principles presented in (Avril et al., 2007).

When $\varepsilon^{* *}$ is used instead of $\varepsilon^{*}$ in equation [12], the remaining error can be used to quantify the quality of the experimental strain reconstruction from the data. The reference value for $v$ is 0.286 .

The obtained values of $v$ for different types of reconstruction (FEA with different mesh sizes, AD with different spans of the weighting function), using noiseless data, are shown in Figure 6(a) It can be noticed that :

- the error increases when the mesh size or the span of the weighting function increases, due to the worsening faithfulness of the reconstruction;

- the curve for DA is smoother than the curve for FEA. Indeed, for a given span of the weighting function, the reconstruction obtained with DA is unique, and so is the identified Poisson's ratio. But for FEA, as many different meshes give as many different reconstruction, hence as many identified Poisson's ratio. This is why the curve for FEA in Figure 6(a) has this serrated aspect.

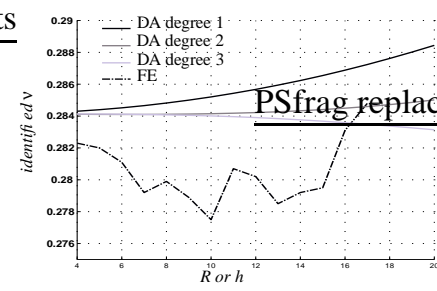

(a) unperturbed measurements

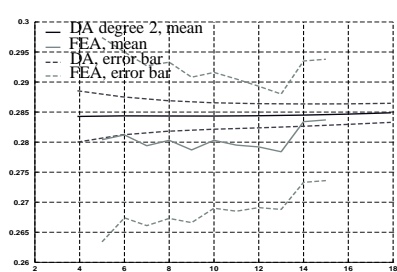

(b) noisy data - mean value and error bar

Figure 6. Identifi ed $v$ as a function of the characteristic size

Identification results obtained with noisy data are shown in Figure 6(b) in terms of mean value and error bar of plus and minus the standard deviation value of the identified Poisson's ratio, deduced from the use of 150 samples of white noise. The average of the identified Poisson's ratio on the samples converges to the noiseless case with the number of samples. First, one can remark that the larger the mesh size or the span of the weighting function, the smaller the standard deviation. For DA, the systematic error illustrated figure 6(a) remains small with respect to the standard deviation and therefore the increase of the span of the weighting function improves the identification accuracy within the studied range. Furthermore, when using the FEA, the main effect is still the large variations of the average value from one mesh size 
to another when using FEA and the dispersion due to the mesh dependence of the reconstruction is of the same range as the one due to the noise, the use of a larger mesh size does not improve the identification results much. In all cases, the identification error remains small. The drawback related to the meshing dependency of the FEA is hence emphasized by the fact that the identification errors remain small.

Eventually, due to the averaging effect of integration in equation [12], it can be concluded that the error about the Poisson's ratio which is identified with the VFM does not constitute a relevant criterion for qualifying the reconstruction approaches. Other criteria based on local errors may be used instead.

\section{Real test data : detection of non-linearities}

In this section, the two reconstruction approaches are applied to data obtained from an open-hole tensile test carried out onto a glass/epoxy laminated plate having a quasi-isotropic stacking sequence : $\left[-45_{4}, 90_{4}, 45_{4}, 0_{4}\right]_{s}$. In such tests, fracture of the $90^{\circ}$ underlying plies occurs quite early. An objective of full-field displacement fields is to detect the onset and spatial location of this fracture. Thirty frames of the displacement fields were measured with the grid methods in a region around the hole of the specimen, for tensile loads ranging from 0 to $13.3 \mathrm{kN}$. It has been proposed in (Pierron et al., 2007) to characterize the fracture process by detecting local non linearities of the response. A first estimation of these non-linearities is obtained by computing the discrepancy between the real strain field and the strain field resulting from the extrapolation of the linear response. One has therefore to determine the latter.

The linear response is estimated from the first ten frames as the slope of the response of each pixel :

$$
\min _{\varepsilon_{\text {lin }}} \sum_{i}^{N_{\text {lin }}}\left(F_{i} \varepsilon_{\text {lin }}-\varepsilon\left(\underline{x}, F_{i}\right)\right)^{2} \quad \text { with, } \quad N_{\text {lin }}=10
$$

Then, it is possible to define the non-linear part of the strain field for a given load $F$ :

$$
\Delta \varepsilon(\underline{x}, F)=F \varepsilon_{l i n}(\underline{x})-\varepsilon(\underline{x}, F)
$$

The $\Delta \varepsilon$ fields reconstructed by the two approaches are shown for three different loads in Figure 7 and 8 The detection of the non-linearities around the hole gives better results than the previous method used in (Pierron et al., 2007), with a sharper zone providing a more precise localization. Both methods yield similar results, even if the FEA approach remains sensitive to the underlying mesh. Nevertheless, the perturbations on the measurements are more severe than in the numerical case and one would have to input more a priori information on the fields to get better results. This leads us to regularization approaches, which are considered as one of the major prospects of this study. 


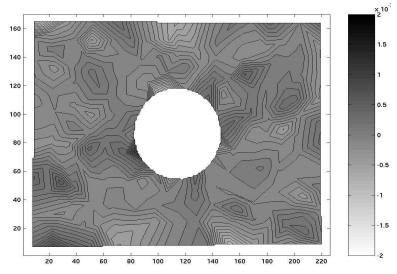

(a) Load $7.5 \mathrm{kN}$

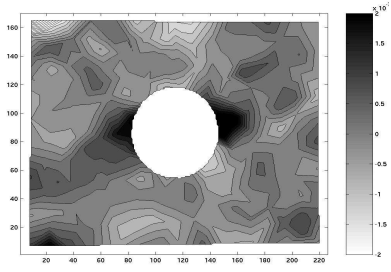

(b) Load $9.7 \mathrm{kN}$

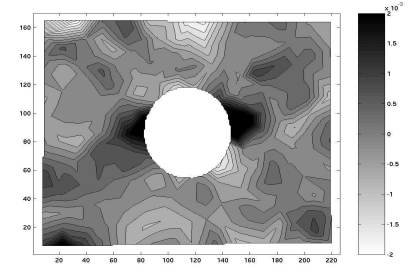

(c) Load $13.3 \mathrm{kN}$

Figure 7. Non-linear part of the displacement fi eld - Finite Elements Approximation

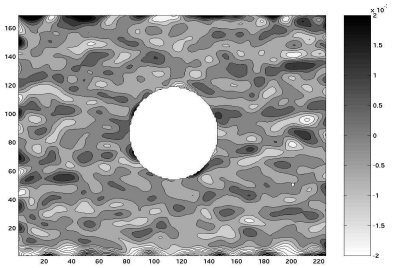

(a) $\operatorname{Load} 7.5 \mathrm{kN}$

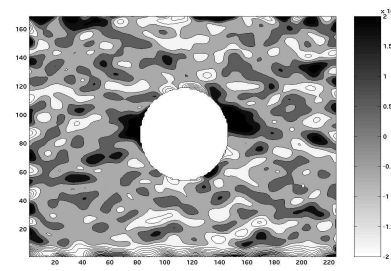

(b) Load $9.7 \mathrm{kN}$

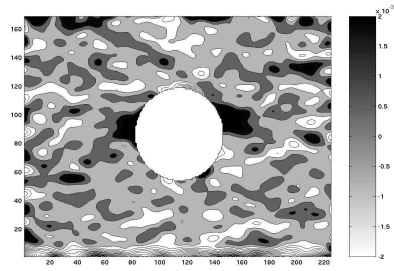

(c) Load $13.3 \mathrm{kN}$

Figure 8. Non-linear part of the displacement fi eld - Diffuse Approximation

\section{Conclusion}

This paper has presented two methods for reconstructing strain fields from fullfield displacement data. The aim of the methods is to handle measurement perturbations and control their filtering. In both methods, the regularization and the precision of the reconstructed fields are controlled by only one parameter, the mesh size or the span of the weighting function. The results on the examples are satisfactory. The FEA approach has a lower computational cost, but provides larger errors in the reconstructed field. Another disadvantage is the multiplicity of the reconstructions for a given mesh size, as two different meshes provide two different reconstructed fields. The DA approach does not have this drawback and offers a richer way of reconstructing the fields, by easing the choice of parameters controlling the errors and letting the possibility of any enrichment of the basis.

It has been shown on a first example that the choice of the mesh size (FEA) or the span of the weighting function (DA) can improve the reconstruction quality. Therefore, it would be interesting to establish a criterium for choosing them as point de- 
pendent functions, leading to size maps for the reconstruction process. Studies about the choice of a relevant criterium are still on-going.

Finally, once the choice of the parameters controlling the errors will be optimized in both approaches, implementation of regularization techniques, such as Tikhonov ones, will be investigated for further improvements.

\section{Bibliographie}

Avril S., Pierron F., « General framework for the identifi cation of constitutive parameters from full-fi eld measurements in linear elasticity », International Journal of Solids and Structures, vol. 44, p. 4978-5002, 2007.

Balaco de Morais A., « Open hole tensile strength of quasi-isotropic laminates », Composites Science and Technology, vol. 60, p. 1997-2004, 2000.

Cleveland W., Loader C., Smoothing by local regression : principles and methods, Springer, 1995.

Feng Z., Rowlands R., « Smoothing fi nite-element and experimental hybrid technique for stress analyzing composites », Computers and Structures, vol. 6, p. 631-639, 1991.

Geers M., De Borst R., Brekelmans W., « Computing strain fi elds from discrete displacement fi elds in 2D solids », International Journal of Solids and Structures, vol. 33, n² 29, p. 42934207, 1996.

Grédiac M., Pierron F., Avril S., Toussaint E., « The virtual fi elds method for extracting constitutive parameters from full-fi eld measurements : a review», Strain, vol. 42, p. 233-253, 2006.

Hickernell F. J., Hon Y. C., « Radial basis function approximations as smoothing splines », Applied Mathematics and Computation, vol. 102, n 1, p. 1-24, 1999.

Kobayashi A., Handbook on Experimental Mechanics, Wiley, 1993.

Lira I., Cordero R., François M., C. V.-E., « The uncertainty of experimental derivarives : application to strain measurement », Measurement Science and technology, vol. 15, p. 23812388, 2004.

Nayroles B., Touzot G., Villon P., « La méthode des éléments diffus », Comptes rendus de l'Académie des Sciences, série 2, Mécanique, Physique, Chimie, Sciences de l'Univers, Sciences de la Terre, vol. 313, n² 2, p. 133-138, 1991.

Pierron F., Green B., Wisnom M. R., « Full-fi eld assessment of the damage process of laminated composite open-hole tensile specimens. Part I : Methodology », Composites Part A : Applied Science and Manufacturing, 2007.

Schreier H., Sutton M., « Systematic errors in digital image correlation due to undermatched subset shape functions », Experimental Mechanics, vol. 42, p. 303-310, 2002.

Surrel Y., « Moiré and grid methods : a signal processing approach. », in J. Pryputniewicz, Ryszard J. et Stupnicki (ed.), Interferometry '94 : Photomechanics, vol. 2342, The International Society for Optical Engineering, SPIE, p. 213-220, Nov, 1994.

Wei T., Li M., « High order numerical derivatives for one-dimensional scattered noisy data », Applied Mathematics and Computation, vol. 175, p. 1744-1759, 2006. 


\section{ANNEXE POUR LE SERVICE FABRICATION \\ A FOURNIR PAR LES AUTEURS AVEC UN EXEMPLAIRE PAPIER \\ DE LEUR ARTICLE ET LE COPYRIGHT SIGNE PAR COURRIER \\ LE FICHIER PDF CORRESPONDANT SERA ENVOYE PAR E-MAIL}

1. ARTICLE POUR LA REVUE :

L'objet. Volume $8-n^{\circ} 2 / 2005$

2. Auteurs :

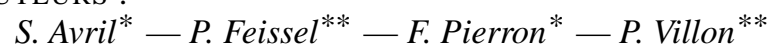

3. TITRE DE L'ARTICLE :

Estimation of the strain fi eld from full-fi eld displacement noisy data

4. TITRE ABRÉGÉ POUR LE HAUT DE PAGE MOINS DE 40 SIGNES :

Strain reconstruction from noisy data

5. DATE DE CETTE VERSiON :

14 septembre 2007

6. COORDONNÉES DES AUTEURS :

- adresse postale :

* Laboratoire de Mécanique et Procédés de Fabrication

Ecole Nationale Supérieure d'Arts et Métiers

BP 508 rue Saint Dominique 51006 CHÂLONS EN CHAMPAGNE

** Laboratoire Roberval de Mécanique, UTC,

BP 20529 rue Personne de Roberval 60205 COMPIEGNE

- téléphone : 0344234604

- télécopie : 0000000000

- e-mail : pierre.feissel@utc.fr

7. LOGICIEL UTILISÉ POUR LA PRÉPARATION DE CET ARTICLE :

LTTE, avec le fichier de style article-hermes.cls,

version $1.23 \mathrm{du} 17 / 11 / 2005$.

8. FORMULAIRE DE COPYRIGHT :

Retourner le formulaire de copyright signé par les auteurs, téléchargé sur :

http: //www.revuesonline.com

SERVICE Éditorial - HERMES-LAVOISIER 14 rue de Provigny, F-94236 Cachan cedex

Tél. : 01-47-40-67-67

E-mail : revues@lavoisier.fr

Serveur web : http://www.revuesonline.com 